\section{LEIS ESTADUAIS COMPLEMENTARES À LEI DE INOVAÇÃO FEDERAL: IMPACTO SOBRE A IMPLEMENTAÇÃO DE INOVAÇÃO NAS INDÚSTRIAS DOS ESTADOS BRASILEIROS}

\author{
STATES' COMPLEMENTARY LAWS TO THE \\ FEDERAL INNOVATION LAW: IMPACT ON THE \\ IMPLEMENTATION OF INNOVATION IN THE \\ INDUSTRIES OF BRAZILIAN STATES
}

\section{RESUMO}

Este estudo tem como objetivo investigar se a adoção de leis de inovação complementares pelos estados impactou a implementação de inovações por parte da indústria local. A pesquisa é descritiva, com a utilização de dados da Pesquisa de Inovação - IBGE (PINTEC), nos triênios de 2000, 2003, 2005, 2008 e 2011. Para a comparação do desempenho inovativo entre estados, é utilizada a técnica de ANOVA para medidas repetidas. Os resultados demonstraram que 12 dos 14 estados apresentaram redução no número de empresas que implementaram inovação entre 2008 e 2011. Evidenciou-se, ainda, que não há diferençasignificativa na implementação de inovações por parte da indústria local nos estados que possuem leis complementares de inovação e aqueles que ainda não as implementaram.

Palavras-chave: Inovação. Lei Complementar. Estados da Federação. Desempenho inovativo.

\begin{abstract}
This study aims to investigate if the adoption of innovation laws at Brazilian states level impacts on the implementation of innovations in the local based industry. The research is descriptive; it uses data from PINTEC - Innovation Survey from IBGE, in 2000 three-year periods, 2eame003, 2005, 2008 and 2011. For comparison of the industrial implementation on innovation at statelevel, ANOVA is used for repeated measures. The results showed that 12 of the 14 states had decreased the implementation of innovation between 2008 and 2011. It was evident, even though there is no significant difference in the implementation of innovation in industry at states that have complementary laws of innovation and
\end{abstract}


those that have not implemented them.

Keywords: Innovation. Complementary Law. Brazilian States. Innovative Performance.

\section{INTRODUÇÃO}

O processo de acirrada concorrência mundial, marcada por disputa pela apropriação da informação, do conhecimento e do desenvolvimento da inovação, indica a necessidade do Brasil de construir um modelo de desenvolvimento tecnológico autônomo (MATIAS-PEREIRA; KRUGLIANSKAS, 2006).

A perspectiva dos sistemas nacionais de inovação é importante porque nenhuma firma inova de maneira isolada, elas inovam por meio das relações entre empresa e outros tipos de organizações, dentro de um contexto institucional (KRETZER, 2009). Dessa forma, é importante entender como muitas políticas públicas influenciam os sistemas de inovação e a economia como um todo, tendo em vista que um conjunto amplo de políticas afeta as estratégias de desenvolvimento social, que, por sua vez, afetam a aprendizagem e a formação de competência (CAMARA; BRASIL, 2015). Isidro Filho e Guimarães (2010) afirmam que existem três vertentes da literatura sobre inovação:

a) inovação quanto ao processo de difusão e adoção de novas tecnologias, envolvendo estudos descritivos em termos de características ou padrões de adoção de inovações em tempo e espaço;

b) inovação na perspectiva dos seus determinantes organizacionais, ou seja, atributos que favorecem ou inibem a inovação e seu desenvolvimento e;

c) a inovação na perspectiva dos processos, em especial, na identificação de suas fases ou estágios.

Este trabalho foca na vertente - inovação quanto aos processos de difusão e adoção de novas tecnologias - apresentando a perspectiva do marco legal como fator determinante no estabelecimento de um ambiente nacional adequado para disseminação da cultura da inovação.
Com base na avaliação da experiência de alguns países bem-sucedidos na implementação de políticas industrial e tecnológica, Matias-Pereira e Kruglianskas (2006) evidenciaram que a aprovação e a regulamentação da lei de inovação representam um ponto de partida importante para fomentar a construção de um modelo de desenvolvimento tecnológico autônomo no Brasil. Os Estados da Federação estão-se mobilizando e elaborando as respectivas leis estaduais de incentivo à inovação dada a necessidade do envolvimento de todas as esferas de governo, federal, estadual e municipal, com esta questão (ARAÚJO, 2007). Destaca-se, ainda, que, conforme o Manual de Oslo (OECD, 2018), as regulações e os padrões bem delineados podem fornecer um forte indício para sustentar e guiar atividades inovadoras.

Considerando a relevância do tema, este artigo tem como foco a lacuna de pesquisa da relação entre a adoção de leis de inovação complementares pelos Estados da Federação e o impacto no desempenho inovativo das empresas locais. Matias-Pereira (2004) ressalta que a elevação do nível de utilização da ciência e tecnologia não ocorre de forma espontânea, razão pela qual tanto os países ricos como os mais pobres necessitam apoiar-se em políticas industriais, com base no desenvolvimento tecnológico, voltadas para o comércio exterior, dirigidas para acelerar os ganhos de competitividade. No trabalho seminal de Stal e Fujino (2005), foi identificado o receio das empresas quanto às reais possibilidades de o governo transformar os atos previstos em lei em ação prática, justamente pela conhecida dificuldade de operar com velocidade adequada às empresas e pela necessidade de uma regulamentação mais extensa.

Assim, após uma revisão sistemática da literatura, apresenta-se a seguinte questão de pesquisa: os Estados da Federação apresentaram significativo crescimento no processo inovativo das empresas locais após a implantação de leis complementares de inovação?

Com base na análise da literatura, apresentam-se as seguintes hipóteses de pesquisa: 
H1. Nos Estados da Federação que implementaram leis complementares à "Lei de Inovação", as indústriaspassaram a apresentar mais inovações que no período anterior.

H2. As indústrias dos estados que possuem leis complementares à "Lei de Inovação" desenvolveram mais inovações que aquelas dos estados que não possuem leis complementares.

A pesquisa é de caráter exploratória, com abordagem positivista. Serão utilizados dados secundários, disponibilizados pelo Ministério do Planejamento, Orçamento e Gestão mediante a publicação de sua pesquisa de Inovação - PINTEC, considerando o período de 2000 a 2011. A análise dos dados será realizada por meio de estatística descritiva, utilizando ANOVA de medidas repetidas.

Este artigo está estruturado em quatro sessões: na primeira sessão, é apresentado o referencial teórico que fundamenta a importância da promulgação de leis específicas para o estimulo da inovação, assim como um abreve apresentação da legislação brasileira; na segunda sessão, são comentados os métodos e procedimentos adotados para coleta de dados e na terceira sessão, são apresentadas a descrição e a análise dos dados obtidos por meio dos estudos estatísticos. As considerações finais foram apresentadas na última sessão.

\section{REFERENCIAL TEÓRICO}

Uma inovação pode ser definida como a implementação de um produto ou serviço novo ou significativamente melhorado, ou um processo, ou um novo método de marketing, ou um novo método organizacional nas práticas de negócios, na organização do local de trabalho ou nas relações externas (OCDE, 2006). O processo de inovação tecnológica decorre da sinergia entre pesquisadores, agentes econômicos, grupos sociais, indivíduos, organismos governamentais e resulta na afluência entre as potencialidades científicas e as efetivas necessidades socioeconômicas do país (MATIAS-PEREIRA, 2003). Por mais que se defina a empresa como o agente que introduz a inovação, e que essa lança novos produtos no mercado e utiliza novos processos de produção ou novos processos organizacionais, entende-se que o desenvolvimento tecnológico não é fruto da ação individualizada das empresas. A inovação e o desenvolvimento tecnológico são produtos da coletividade. É a interação entre vários agentes econômicos que produz o desenvolvimento tecnológico (STAUB, 2001).

Em seus trabalhos semanais da segunda década do século XX, Schumpeter (1961) já afirmava que o impulso fundamental que põe e mantém em funcionamento o sistema capitalista deve-se a novos bens de consumo, novos métodos de produção ou transportes, novos mercados e novas formas de organização industrial criadas pela empresa capitalista. A economia contemporânea se move em função da geração e incorporação de inovações. Com feito, inovar tornou-se a principal arma de competição entre empresas e entre países. $\mathrm{Na}$ atualidade, deter conhecimento tecnológico conduz à dominação econômica e política (STAUB, 2001).

Araújo (2007) argumenta que a inovação é muito mais do que um conceito ou uma prática, é uma necessidade, uma postura de atuação diante da necessidade de desenvolvimento do país; frisa ainda que é pacífico o entendimento sobre a importância do sistema de inovação como mecanismo essencial para o desenvolvimento social, tecnológico e econômico. Freeman e Soete (2008) evidenciam, ainda, a importância crucial da acumulação tecnológica por meio de uma combinação de importação de tecnologias com atividades locais e políticas intervencionistas proativas para fomentar indústrias nascentes.

No Brasil, segundo Stal e Fujino (2005), há uma carência tanto de investimento em pesquisa como a inexistência de uma cultura para a inovação, o que decorre da carência de políticas de incentivo a atividades de pesquisa e desenvolvimento tecnológico. Dessa forma, o Brasil, segundo Carvalho (2009), está aquém de seu potencial de inovação, considerando diversos indicadores e que, mesmo com esforços para fomentar a inovação no país com o au- 
mento do número de incubadoras, a indústria de base tecnológica brasileira encontra-se ainda concentrada no sul e sudeste do país. No caso das economias em desenvolvimento, políticas nacionais de promoção do desenvolvimento industrial e de ciência, tecnologia e inovação são condição indispensável a uma inserção menos subordinada no cenário internacional (STAUB, 2001). Conforme autores evolucionários seminais (FREEMAN; SOETE, 2008), o ambiente nacional pode ter considerável influência para estimular, facilitar, retardar ou impedir as atividades inovativas das firmas. Estudos como os de Hölzl e Janger (2014) e Silva Filho, Braga e Rebouças (2017) expõem a situação dessas barreiras nas empresas em diversos contextos.

Fonseca (2010) afirma que o principal papel do governo no que concerne à inovação tecnológica, portanto, é o de prover os incentivos corretos ao desenvolvimento e à difusão de ideias por parte do setor privado (ações indiretas). Promover um ambiente político, econômico e institucional que estimule as empresas a investir em ciência, tecnologia, pesquisa e desenvolvimento. Guellec (2001) destaca, nesse sentido, que as políticas de apoio à inovação tecnológica podem contar com um conjunto diversificado de instrumentos, como patentes, concessões, contratos, incentivos fiscais ou pesquisa pública. Mas, de acordo com Cooter et al. (2011), pouca atenção tem sido dada pelos economistas e legisladores para as instituições, leis e sistemas jurídicos; em particular, aqueles são mais adequados para promover a inovação e o crescimento econômico. Destacam, ainda que os legisladores que projetam e implementam o sistema normativo devem começar a considerar, seriamente, os efeitos da lei sobre a inovação e o crescimento.

Os objetivos regulatórios do século XXI são muito complexos, pois não visam apenas restringir o poder do monopólio - forte objetivo das leis dos séculos pós-revolução industrial, mas também quer promover o crescimento econômico e inovação para alcançar um conjunto diversificado de objetivos públicos e privados (HADFIELD, 2011). Além disso, um claro re- conhecimento do impacto econômico da política jurídica é essencial para a produção da infraestrutura jurídica necessária para promover a inovação e o crescimento em uma economia global.

A inovação tecnológica afeta a maioria dos mecanismos econômicos, pois ela está no centro da estratégia das empresas, nas estruturas de mercado, no crescimento das nações, nas mudanças na demanda de trabalho e distribuição de renda. Diversos países têm feito da inovação uma prioridade em suas políticas econômicas (GUELLEC, 1999).

O Ministério da Ciência Tecnologia e Inovação do Brasil (MCTI) destaca que desafio de se estabelecer no país uma cultura de inovação está amparado na constatação de que a produção de conhecimento e a inovação tecnológica passaram a ditar, crescentemente, as políticas de desenvolvimento dos países (BRASIL, 2013).

Acrescenta-se que os preceitos constitucionais estabelecidos no artigo 218 da Constituição Federal estabelece que o Estado promoverá e incentivará o desenvolvimento científico, a pesquisa e a capacitação tecnológicas (BRASIL, 1988).

Os pressupostos que fundamentam a criação de um arcabouço jurídico para a questão da inovação tecnológica nacional, segundo Araújo (2007), são:

a) reconhecimento da inovação tecnológica como um dos fatores de desenvolvimento do país e de sua inserção no sistema econômico globalizado;

b) recuperação do gap tecnológico do país;

c) estímulo à criação de um sistema de inventiva nacional com envolvimento de todos os atores da sociedade.

A Lei $n^{0} 10.973$, de 2 de dezembro de 2004 (10973/04), denominada "Lei da Inovação", reflete a necessidade de o país contar com dispositivos legais eficientes que contribuam para o delineamento de um cenário favorável ao desenvolvimento científico, tecnológico e ao incentivo à inovação (BRASIL, 2013)

Garnica e Jugend (2009) destacam ainda que a Lei 10.973/04, regulamentada em outu- 
bro de 2005, representa, contemporaneamente, a mais importante diretriz governamental para as atividades cooperativas entre os agentes do sistema nacional de inovação brasileira.

Corroborando os autores, Moreira et al. (2007) destacam ainda que a Lei de Inovação é considerada um dos principais pontos de referência da Política Industrial, Tecnológica e de Comércio Exterior (Pitce).

O marco regulatório brasileiro (Lei 10.973/04) está organizado em torno de três vertentes conforme identificado no quadro 1.

Quadro 1 - Vertentes da Lei de Incentivo a Inovação N¹0.973/2004

\begin{tabular}{|c|c|c|}
\hline VERTENTE I & VERTENTE II & VERTENTE III \\
\hline $\begin{array}{l}\text { Constituição de ambiente pro- } \\
\text { pício às parcerias estratégicas } \\
\text { entre as universidades, institu- } \\
\text { tos tecnológicos e empresas. }\end{array}$ & $\begin{array}{l}\text { Estimulo à participação de } \\
\text { instituições de ciência e tec- } \\
\text { nologia no processo de ino- } \\
\text { vação. }\end{array}$ & $\begin{array}{l}\text { Incentivo à inovação na em- } \\
\text { presa. }\end{array}$ \\
\hline $\begin{array}{l}\text { 1. Contempla diversos meca- } \\
\text { nismos de apoio e estímulo à } \\
\text { constituição dealianças estra- } \\
\text { tégicas e ao desenvolvimento } \\
\text { de projetos cooperativos entre } \\
\text { universidades, institutos tec- } \\
\text { nológicos e empresas nacio- } \\
\text { nais. } \\
\text { 2. Facilidades para que as ins- } \\
\text { tituições de ciência e tecnolo- } \\
\text { gia (ICT)possam comparti- } \\
\text { lhar, mediante remuneração, } \\
\text { seus laboratórios, instalações, } \\
\text { infra-estrutura e recursos hu- } \\
\text { manos com empresas, para } \\
\text { atividades de pesquisa con- } \\
\text { forme a situação especificada } \\
\text { na lei. }\end{array}$ & $\begin{array}{l}\text { 1. Faculta às ICT(s) celebrar } \\
\text { contratos de transferência de } \\
\text { tecnologia e de licenciamento } \\
\text { de patentes de sua propriedade, } \\
\text { prestar serviços de consultoria } \\
\text { especializada em atividades de- } \\
\text { senvolvidas no âmbito do setor } \\
\text { produtivo, assim com estimular } \\
\text { a participação de seus funcioná- } \\
\text { rios em projetos em que a ino- } \\
\text { vação seja o principal foco. } \\
\text { 2. Determina que cada ICT, cons- } \\
\text { titua um Núcleo de Inovação } \\
\text { Tecnológica (NIT) próprio ou em } \\
\text { associação com outras ICT. } \\
\text { 3. Os pesquisadores vincula- } \\
\text { dos as ICT, quando envolvidos } \\
\text { nas atividades de prestação de } \\
\text { serviços empreendidas por } \\
\text { suas instituições, poderão, em } \\
\text { casos específicos, beneficiar- } \\
\text {-se do resultado financeiro dos } \\
\text { serviços prestados. } \\
\text { 4. Faculta os servidores pú- } \\
\text { blicos das ICT(s), a receber, } \\
\text { como estímulo à inovação, } \\
\text { bolsa diretamente de institui- } \\
\text { ção de apoio ou de agência de } \\
\text { fomento. }\end{array}$ & $\begin{array}{l}\text { 1. Estimula uma maior contri- } \\
\text { buição do setor produtivo em } \\
\text { relação à alocação de recurso } \\
\text { financeiro na promoção da } \\
\text { inovação. } \\
\text { 2. Prevê a concessão, por par- } \\
\text { te da União, das ICT(s) e das } \\
\text { agências de fomento, de re- } \\
\text { cursos financeiros, humanos, } \\
\text { materiais ou de infraestru- } \\
\text { tura, para atender às empre- } \\
\text { sas nacionais envolvidas em } \\
\text { atividades de pesquisa e de- } \\
\text { senvolvimento. Os recursos } \\
\text { financeiros poderão vir sob a } \\
\text { forma de subvenção econômi- } \\
\text { ca, financiamento ou partici- } \\
\text { pação societária. } \\
\text { 3. Contempla apoio à realização } \\
\text { de atividades de pesquisa e de- } \\
\text { senvolvimento, que envolvam } \\
\text { risco tecnológico, para solução } \\
\text { de problema técnico específico } \\
\text { ou obtenção de produto ou pro- } \\
\text { cesso inovador, assim como a } \\
\text { implementação pelas agências } \\
\text { de fomento, de programas com } \\
\text { ações dirigidas especialmente à } \\
\text { promoção da inovação nas mi- } \\
\text { cro e pequenas empresas. }\end{array}$ \\
\hline
\end{tabular}

Fonte: (BRASIL, 2013). 
Na vertente III, que se espera das leis de incentivo à inovação que também haja um "incentivo" à inovação nas empresas, isto é, que, com a implantação destas leis, tenha-se um aumento de inovações na indústria.

Matias-Pereira e Kruglianskas (2006), com base em um estudo empírico, que realizou 19 entrevistas abertas com os principais atores dos principais segmentos envolvidos na construção da Lei da Inovação, apresentam suas características no quadro 2.

Quadro 2 - Avaliação da Lei da Inovação e suas características

a) A partir da lei e de sua regulamentação, abrem-se novas possibilidades para a constituição de fundos, criação de sociedades anônimas, tomada de empréstimos, repasse de incentivos.

b) A lei tende a contribuir para consolidar a relação produção, pesquisa e União, visto que busca desenvolver esforços de diversos ministérios e da sociedade.

c) Identifica-se a necessidade de integrar a Lei de Inovação à nova Política Industrial e sua ação coordenada às políticas estaduais e municipais.

d) É necessário existir um arcabouço institucional consistente, a partir de uma política industrial e tecnológica consistente, que permita que os dirigentes de empresas globais possam confiar a brasileiros a gestão de um centro de tecnologia.

e) Há a necessidade de adoção de medidas para que as despesas de P\&D sejam deduzidas no lucro tributável.

f) Necessidade de desoneração dos investimentos de P\&D; g) necessidade de definição de incentivos diretos para a formação dos empresários em knowhow empresarial, estratégias de marketing, diferenciação competitiva e princípios de $\mathrm{P} \& \mathrm{D}$;

g) Necessidade de oferta de incentivos diretos para a formação da infraestrutura de P\&D.

h) Necessidade de financiamentos privilegiados de pesquisa para projetos envolvendo universidade-indústria.

i) Necessidade de apoio financeiro e técnico-jurídico para patenteamento de novas descobertas realizadas no Brasil e no exterior.

j) A lei é uma sinalização clara de que o governo federal está orientando medidas para a geração de estímulos à inovação, flexibilizando atividades e relações das instituições científicas e tecnológicas.

1) Falta de definições claras para a geração de estímulos e apoio à micro e pequenas empresas do país.

m) Falhas na forma de definição das normas que tratam da interação entre os três atores principais para o sucesso da lei de inovação, ou seja, o inventor, a universidade e os institutos de pesquisa, e o capital de risco;

n) Não se preveem mecanismos que permitam às universidades controlar adequadamente o trabalho dos pesquisadores que se afastarem para desenvolver projetos de pesquisa no setor privado.

o) Necessidade de maior preocupação principalmente com a transparência na relação entre os professores das universidades e empresas.

p) A lei é carente de normas definidoras que permitam às universidades controlar melhor o trabalho dos pesquisadores que venham a se afastar do ambiente acadêmico para desenvolver projetos no setor privado. Para esses entrevistados, é indesejável para a sociedade que o interesse do pesquisador prevaleça em relação ao da instituição a que ele está ligado.

q) O risco do interesse do pesquisador acabar prevalecendo em relação ao da instituição a que ele está ligado.

r) A lei de inovação não levou em consideração a essencialidade de as empresas desenvolverem seus próprios sistemas de aprendizado tecnológico e organizacional - precisam criar departamentos específicos de P\&D.

Fonte: (MATIAS-PEREIRA; KRUGLIANSKAS, 2006).

Considerando os resultados de sua pesquisa, Matias-Pereira e Kruglianskas (2006) questionam se a lei de inovação - aceita como parte essencial do arcabouço institucional para fortalecer 
as áreas de pesquisa e da produção de conhecimento no Brasil -, poderá fomentar, adequadamente, a criação de novos ambientes, propícios à geração e absorção de inovações, atuando como ferramenta de apoio à Política Industrial e Tecnológica do Brasil.

É fundamental a participação do estado como o indutor do processo de transformação, inserindo, em suas principais preocupações, a busca de inovação e de modernização dos setores da economia (SICSÚ; LIMA, 2001).

A grande tarefa é articular os instrumentos federais e estaduais com as instituições locais, seja como sistema produtivo e empresarial, seja como instituições públicas e civis de cada localidade. Essa tarefa exige um esforço permanente e passa pela criação ou fortalecimento de instâncias coordenadoras locais, agências locais de desenvolvimento, sindicatos, associações empresariais ou outras formas de coordenação local. Como cada localidade ou região possui características próprias, não há como sugerir ou implementar estruturas únicas e uniformes (DINIZ, 2001).

Os estados da federação têm adotado Leis Complementares à Lei de Inovação a fim de estabelecer um marco regulatório regional que possibilite o desenvolvimento de inovação no contexto local. O Senado Federal (BRASIL, 2013) destaca que as Leis Complementares diferem das Leis Ordinárias por exigirem o voto da maioria dos parlamentares que compõe a Câmara dos Deputados e o Senado Federal para serem aprovadas. E, somente devem ser adotadas para regulamentar assuntos específicos, quando expressamente determinado na Constituição da República.

O sucesso dessas iniciativas passa a depender da capacidade local de implementar tais estratégias. O processo de inovação temforte vinculação regional ou local.

Segundo Diniz (2001) comocada localidade ou região possui características próprias,não há comosugerir ou implementar estruturas únicas e uniformes.

O sucesso dessas iniciativas passa a depender da capacidade local de implementar tais estratégias. Nessa perspectiva, a complementaridade entre as políticas e os instrumentos federais e de cada estado deve ser vista comouma tarefa permanente e recorrente, com ações horizontais e verticais. Cada estado deveria ser estimulado a organizar o sistema estadual e ciência e tecnologia, que serviria de interface na articulação vertical entre o Governo Federal e o governo de cada Estado (DINIZ, 2001).

\section{METODOLOGIA}

Faria (2012) afirma que a dimensão epistemológica é a única garantia de coerência na produção e no desenvolvimento do conhecimento e que, portanto, é a partir dessa instância que se estabelece o diálogo entre teorias, entre disciplinas e entre ciências.

Dessa forma, esta pesquisa enquadra-se no paradigma positivista, pois visa buscar evidências encontradas com o emprego de proposições formais, testar hipóteses e fazer inferências sobre um fenômeno a partir de uma amostrada população (BARBOSA, 2012).

Foi realizada uma pesquisa descritiva, cujo objetivo é identificar e obter informações sobre as características de um determinado problema ou questão (COLLIS; HUSSEY, 2005).

Com base na análise da literatura, apresentam-se a seguintes hipóteses de pesquisa:

H1. Nos Estados da Federação que implementaram leis complementares à Lei de Inovação, as indústrias passaram a apresentar mais inovações que no período anterior.

H2. As indústrias dos estados que possuem Leis complementares à Lei de Inovação Tecnológica desenvolveram mais inovações que aquelas dos estados que não possuem leis complementares.

São utilizados dados secundários, disponibilizados pelo Ministério do Planejamento, Orçamento e Gestão (assim como pelo IBGE) por meio da publicação de sua pesquisa de Inovação - PINTEC, considerando o período de 2000 a 2011, ou seja, as publicações de 2000, 2003, 2005, 2008 e 2011 (IBGE, 2019). 
Utilizam-se, especificamente, os dados da tabela 2.1 do PINTEC - Variáveis selecionadas das empresas das indústrias extrativa e de transformação, segundo as grandes regiões e unidades da Federação selecionadas - Brasil.

A análise dos dados foi realizada mediante comparação do número de empresas que implantaram inovação de produtos e/ou processo nos estados selecionados, considerando os resultados do PINTEC um período antes e um, após a implantação de leis complementares de inovação. Não foram consideradas as inovações organizacionais e/ou de marketing.

Nessa primeira fase da análise, que busca obter dados para estudar a hipótese $\mathrm{H} 1$, os dados serão analisados em termos de proporção, ou seja, ano a ano, a dimensão de empresas que implementaram inovação de produto e/ou processo, com base no total de empresas pes-

quisadas do estado no período.

Para comparação do desempenho inovativo entre estados que implementaram leis complementares de inovação e estados que não implementaram, será utilizada a técnica de ANOVA para medidas repetidas, considerando apenas para efeito de comparação, estados da mesma região.

\section{APRESENTAÇÃO E DISCUSSÃO DOS RESULTADOS}

Conforme dados do Ministério da Ciência, Tecnologia e Inovação (2013), o Brasil possui 16 estados com leis complementares à Lei de Inovação Federal de inovação aprovadas, conforme pode ser identificado no quadro 3.

Quadro 3 - Estado que aprovaram leis complementares à Lei de Inovação federal

\begin{tabular}{|c|c|c|c|c|c|}
\hline $\begin{array}{c}\text { Ano de } \\
\text { Implementação }\end{array}$ & 2006 & 2008 & 2009 & 2010 & 2011 \\
\hline $\begin{array}{l}\text { Estado da } \\
\text { Federação }\end{array}$ & Amazonas & $\begin{array}{l}\text { Mato Grosso } \\
\text { Santa Catarina } \\
\text { Minas Gerais } \\
\text { São Paulo } \\
\text { Ceará } \\
\text { Pernambuco } \\
\text { Rio de Janeiro } \\
\text { Bahia }\end{array}$ & $\begin{array}{l}\text { Alagoas } \\
\text { Rio Grande do } \\
\text { Sul } \\
\text { Sergipe } \\
\text { Espírito Santo }\end{array}$ & $\begin{array}{l}\text { Goiás } \\
\text { Mato Grosso } \\
\text { do Sul }\end{array}$ & Tocantins \\
\hline
\end{tabular}

Fonte: (BRASIL, 2013).

Os Estados da Federação implantaram leis de inovação no período de 2006 a 2011, visando dispor sobre incentivos à pesquisa científica e tecnológica e à inovação no ambiente produtivo do estado. Identifica-se, ainda, que a maioria dos estados desenvolveu leis complementares à lei de inovação no ano de 2008 e 2009, quatro anos após a aprovação da Lei Inovação Nacional $\mathrm{n}^{\mathrm{o}} 10.973$, de 2 de dezembro de 2004. O Estado do Amazonas foi pioneiro na implementação de lei estadual complementar, desenvolvendo lei específica para o estado em 2006.

No país, até 2011, de acordo com dados da MCTI, 11 Estados da Federação ainda não possuíam leis complementares de inovação para incentivo à inovação em âmbito local, os quais se listam por região: a) Norte: Acre, Amapá, Pará, Rondônia, Roraima; b) na Região Nordeste: Maranhão, Paraíba, Piauí, Rio Grande do Norte; c) no Centro-Oeste: Distrito Federal e Mato Grosso do Sul.

A Pesquisa de Inovação (PINTEC) é realizada pelo Instituto Brasileiro de Geografia e Estatística (IBGE), com o apoio da Financiadora de Estudos e Projetos - FINEP e do Ministério da Ciência, Tecnologia e Inovação. Em seus dados de 2000 a 2011, considerando os resultados regionais - grandes regiões - apresenta-se o desempenho das regiões Norte, Nordeste, Centro-Oeste, Sudeste e Sul (IBGE, 2016). 
As unidades da Federação selecionadas em todas as edições são: Amazonas, Pará, Ceará, Pernambuco, Bahia, Minas Gerais, Espírito Santo, Rio de Janeiro, São Paulo, Paraná, Santa Catarina, Rio Grande do Sul e Goiás. Contudo, na última edição da pesquisa, em 2011, são apresentados os resultados do estado do Mato Grosso - Região Centro-Oeste.

Desse modo, das 14 unidades da Federação selecionadas no PINTEC, 11 possuem leis complementares de inovação e dois não possuem: Pará e Paraná e mesmo não havendo leis complementares de inovação nestes estados, eles possuem desempenho inovativo significativo para efeito de participação da pesquisa do IBGE.

Observa-se, também, que os Estados de Alagoas, Sergipe e Tocantins, apesar de possuírem leis complementares de inovação, não apresentam desempenho inovativo local representativo para inclusão nos resultados do PINTEC.

A tabela 1 apresenta do número de empresas que inovaram, conformedados do PINTEC nas edições de 2000, 2003, 2005, 2008 e 2011 (IBGE, 2016).

Tabela 1 - Número de empresas que implementaram inovação de produtos e/ ou processos

\begin{tabular}{|c|c|c|c|c|c|c|c|c|c|c|c|}
\hline $\begin{array}{l}\text { Unidade da } \\
\text { Federação }\end{array}$ & Com & $\begin{array}{l}\text { ei } \\
\text { mentar/ } \\
\text { no }\end{array}$ & 2000 & 2003 & $\begin{array}{c}\text { Cresc. } \\
\%\end{array}$ & 2005 & $\begin{array}{c}\text { Cresc. } \\
\%\end{array}$ & 2008 & $\begin{array}{c}\text { Cresc. } \\
\%\end{array}$ & 2011 & $\begin{array}{c}\text { Cresc. } \\
\%\end{array}$ \\
\hline Amazonas & $\operatorname{sim}$ & 2006 & 225 & 203 & $-10 \%$ & 296 & $46 \%$ & 449 & $52 \%$ & 457 & $2 \%$ \\
\hline Pará & não & - & 124 & 378 & $205 \%$ & 440 & $16 \%$ & 433 & $-2 \%$ & 360 & $-17 \%$ \\
\hline Ceará & $\operatorname{sim}$ & 2008 & 511 & 603 & $18 \%$ & 521 & $-14 \%$ & 840 & $61 \%$ & 1104 & $31 \%$ \\
\hline Pernambuco & $\operatorname{sim}$ & 2008 & 485 & 485 & $0 \%$ & 269 & $-45 \%$ & 729 & $171 \%$ & 1052 & $44 \%$ \\
\hline Bahia & $\operatorname{sim}$ & 2008 & 461 & 641 & $39 \%$ & 633 & $-1 \%$ & 1083 & $71 \%$ & 1084 & $0 \%$ \\
\hline $\begin{array}{l}\text { Minas } \\
\text { Gerais }\end{array}$ & $\operatorname{sim}$ & 2008 & 2303 & 3503 & $52 \%$ & 3203 & $-9 \%$ & 5208 & $63 \%$ & 5841 & $12 \%$ \\
\hline $\begin{array}{l}\text { Espírito } \\
\text { Santo } \\
\end{array}$ & $\operatorname{sim}$ & 2009 & 468 & 645 & $38 \%$ & 742 & $15 \%$ & 953 & $28 \%$ & 641 & $-33 \%$ \\
\hline $\begin{array}{l}\text { Rio de } \\
\text { Janeiro }\end{array}$ & $\operatorname{sim}$ & 2008 & 1212 & 1367 & $13 \%$ & 1362 & $0 \%$ & 1713 & $26 \%$ & 1623 & $-5 \%$ \\
\hline São Paulo & $\operatorname{sim}$ & 2008 & 8663 & 9209 & $6 \%$ & 10734 & $17 \%$ & 12379 & $15 \%$ & 12984 & $5 \%$ \\
\hline Paraná & não & - & 1890 & 2607 & $38 \%$ & 3154 & $21 \%$ & 3641 & $15 \%$ & 3432 & $-6 \%$ \\
\hline $\begin{array}{l}\text { Santa } \\
\text { Catarina } \\
\end{array}$ & $\operatorname{sim}$ & 2008 & 2045 & 2480 & $21 \%$ & 2648 & $7 \%$ & 3209 & $21 \%$ & 3555 & $11 \%$ \\
\hline $\begin{array}{l}\text { Rio Grande } \\
\text { do Sul } \\
\end{array}$ & $\operatorname{sim}$ & 2009 & 2413 & 3304 & $37 \%$ & 3225 & $-2 \%$ & 4029 & $25 \%$ & 4627 & $15 \%$ \\
\hline $\begin{array}{l}\text { Mato } \\
\text { Grosso }\end{array}$ & $\operatorname{sim}$ & 2008 & 0 & 0 & - & 0 & - & 0 & - & 254 & - \\
\hline Goiás & $\operatorname{sim}$ & 2008 & 464 & 737 & $59 \%$ & 642 & $-13 \%$ & 1261 & $96 \%$ & 1644 & $30 \%$ \\
\hline Brasil & & & 22698 & 28036 & $24 \%$ & 30377 & $8 \%$ & 38299 & $26 \%$ & 41470 & $8 \%$ \\
\hline
\end{tabular}

Fonte: elaborado pelos autores com base nos dados do PINTEC (IBGE, 2016).

Em números absolutos, identifica-se que o Brasil teve um crescimento nos triênios de 2003 e 2008, visto que apresentou o maior crescimento de empresas, implantando inovação de processos e/ou produtos.

Ao realizar um comparativo por Estado da Federação, um período antes e após a implantação da Lei Complementar de Inovação, percebe-se que os estados do Espírito Santo e Rio de Janeiro tiveram queda no número de empresas que inovaram após a implementação das leis estaduais. E o Estado da Bahia permaneceu 
com o mesmo desempenho após a implantação da Lei de Inovação. Os demais estados apresentaram crescimento no número de empresas que implementaram inovação, em destaque para os estados do Ceará, Pernambuco, Minas Gerais Santa Catarina, Rio Grande do Sul e Minas Gerais, que tiveram um crescimento percentual maior que o nacional, em especial o Ceará, Pernambuco e Goiás, com crescimento acima de $30 \%$.

Ambos os estados que não possuem leis complementares de inovação, Pará e Paraná ti- veram uma queda no número de empresas que inovaram no que se refere aos resultados do PINTEC 2011. Destaca-se, contudo, o desempenho que o estado do Pará tem apresentado em inovação, com diminuição do número de empresas que inovam, em duas edições consecutivas do PINTEC, 2008 e 2011.

$\mathrm{Na}$ tabela2, apresentam-se os dados do PINTEC por unidade da Federação no período de 2000 a 2011, considerando as empresas 1ocais participantes da pesquisa no período.

Tabela 2 - Representatividade das empresas que implementaram inovação de produto e/ou processo em relação às empresas pesquisadas no período

\begin{tabular}{|c|c|c|c|c|c|c|c|}
\hline \multirow{3}{*}{$\begin{array}{c}\text { Unidade da } \\
\text { Federação }\end{array}$} & \multirow{3}{*}{\multicolumn{2}{|c|}{$\begin{array}{l}\text { Lei Complementar/ } \\
\text { Ano } \\
2000\end{array}$}} & \multirow{2}{*}{\multicolumn{5}{|c|}{$\begin{array}{l}\begin{array}{c}\text { Empresas que implementaram inovação de produto } \\
\text { e/ou processo }\end{array} \\
\frac{\text { total empresas pesquisadas no estado }}{}\end{array}$}} \\
\hline & & & & & & & \\
\hline & & & 2003 & 2005 & 2008 & 2011 & \\
\hline Amazonas & $\operatorname{sim}$ & 2006 & 0,53 & 0,38 & 0,51 & 0,61 & 0,40 \\
\hline Pará & não & - & 0,17 & 0,34 & 0,34 & 0,27 & 0,26 \\
\hline Ceará & $\operatorname{sim}$ & 2008 & 0,35 & 0,34 & 0,26 & 0,40 & 0,36 \\
\hline Pernambuco & $\operatorname{sim}$ & 2008 & 0,34 & 0,29 & 0,14 & 0,32 & $\mathbf{0 , 3 5} \uparrow$ \\
\hline Bahia & $\operatorname{sim}$ & 2008 & 0,31 & 0,33 & 0,29 & 0,37 & 0,34 \\
\hline Minas Gerais & $\operatorname{sim}$ & 2008 & 0,28 & 0,35 & 0,29 & 0,41 & 0,40 \\
\hline Espírito Santo & $\operatorname{sim}$ & 2009 & 0,24 & 0,36 & 0,38 & 0,36 & 0,28 \\
\hline Rio de Janeiro & $\operatorname{sim}$ & 2008 & 0,26 & 0,25 & 0,26 & 0,33 & 0,30 \\
\hline São Paulo & $\operatorname{sim}$ & 2008 & 0,33 & 0,31 & 0,34 & 0,36 & 0,33 \\
\hline Paraná & não & - & 0,31 & 0,37 & 0,40 & 0,43 & 0,34 \\
\hline Santa Catarina & $\operatorname{sim}$ & 2008 & 0,39 & 0,36 & 0,35 & 0,38 & 0,35 \\
\hline Rio Grande do Sul & $\operatorname{sim}$ & 2009 & 0,33 & 0,40 & 0,36 & 0,44 & 0,42 \\
\hline Mato Grosso & $\operatorname{sim}$ & 2008 & - & - & - & - & 0,21 \\
\hline Goiás & $\operatorname{sim}$ & 2008 & 0,33 & 0,33 & 0,27 & 0,38 & $\mathbf{0 , 4 7 \uparrow}$ \\
\hline
\end{tabular}

Fonte: elaborado pelos autores com base nos dados do PINTEC (IBGE, 2016).

Considerando os dados apresentados na tabela2, percebe-se que, de maneira geral, os estados apresentaram uma diminuição do número de empresas que inovaram em relação às empresas pesquisadas no âmbito regional. Destacam-se os seguintes Estados, que representaram exceção, apresentando crescimento no triênio de 2009 a 2011: Pernambuco e Goiás.
Ainda analisando os dados apresentados pela tabela 2, identifica-se que apenas os estados do Amazonas, Pernambuco e Goiás apresentaram melhor desempenho após a implementação de leis complementares de inovação, não sendo suficiente para comprovar a hipótese H1, que afirma que, nos estados que implementaram leis complementares à lei de inovação, 
as indústrias passaram a apresentar mais inovações que no período anterior.

Quanto à representatividade do estado, em relação ao total de empresas que inovaram em todo país, apresentam-se os resultados, conforme tabela 3:

Tabela 3 - Implementação industrial de Inovação nos Estados em Relação ao Desempenho Nacional

\begin{tabular}{|c|c|c|c|c|c|c|c|}
\hline \multirow[t]{2}{*}{$\begin{array}{c}\text { Unidade da } \\
\text { Federação }\end{array}$} & \multirow{2}{*}{\multicolumn{2}{|c|}{$\begin{array}{c}\text { Lei Complementar } \\
/ \\
\text { Ano } \\
2000\end{array}$}} & \multicolumn{5}{|c|}{$\begin{array}{l}\begin{array}{l}\text { Empresas que implementaram inovação de produto } \\
\text { e/ou processo }\end{array} \\
\frac{\text { total empresas pesquisadas no estado }}{}\end{array}$} \\
\hline & & & 2003 & 2005 & 2008 & 2011 & \\
\hline Amazonas & $\operatorname{sim}$ & 2006 & 0,010 & 0,007 & 0,010 & 0,012 & $0,011 \downarrow$ \\
\hline Pará & não & - & 0,005 & 0,013 & 0,014 & 0,011 & $0,009 \downarrow$ \\
\hline Ceará & $\operatorname{sim}$ & 2008 & 0,023 & 0,022 & 0,017 & 0,022 & 0,027 \\
\hline Pernambuco & $\operatorname{sim}$ & 2008 & 0,021 & 0,017 & 0,009 & 0,019 & 0,025 \\
\hline Bahia & $\operatorname{sim}$ & 2008 & 0,020 & 0,023 & 0,021 & 0,028 & $0,026 \downarrow$ \\
\hline Minas Gerais & $\operatorname{sim}$ & 2008 & 0,101 & 0,125 & 0,105 & 0,136 & 0,141 \\
\hline Espírito Santo & $\operatorname{sim}$ & 2009 & 0,021 & 0,023 & 0,024 & 0,025 & $0,015 \downarrow$ \\
\hline Rio de Janeiro & $\operatorname{sim}$ & 2008 & 0,053 & 0,049 & 0,045 & 0,045 & $0,039 \downarrow$ \\
\hline São Paulo & $\operatorname{sim}$ & 2008 & 0,382 & 0,328 & 0,353 & 0,323 & $0,313 \downarrow$ \\
\hline Paraná & não & - & 0,083 & 0,093 & 0,104 & 0,095 & $0,083 \downarrow$ \\
\hline Santa Catarina & $\operatorname{sim}$ & 2008 & 0,090 & 0,088 & 0,087 & 0,084 & $0,086 \downarrow$ \\
\hline Rio Grande do Sul & $\operatorname{sim}$ & 2009 & 0,106 & 0,118 & 0,106 & 0,105 & 0,112 \\
\hline Mato Grosso & $\operatorname{sim}$ & 2008 & - & - & - & - & 0,006 \\
\hline Goiás & $\operatorname{sim}$ & 2008 & 0,020 & 0,026 & 0,021 & 0,033 & 0,040 \\
\hline
\end{tabular}

Fonte: elaborado pelos autores com base nos dados do PINTEC (IBGE, 2016).

Dos 14 estados que compõem as unidades da Federação selecionadas para a pesquisa, percebe-se que 8 apresentaram diminuição da representatividade de empresas que implementaram inovação de produtos e processos em relação ao País: Amazonas, Pará, Bahia, Espírito
Santo, Rio de Janeiro, São Paulo, Paraná e Santa Catarina.

Destaca-se, contudo, que os estados do Ceará, Pernambuco, Minas Gerais Rio Grande do Sul e Goiás passaram a ter maior representatividade de inovação no período de 2008 a 2011, 
tendo estes estados implementado leis complementares de inovação entre 2008 e 2009.

Percebe-se, ainda, que os estados que mais têm contribuído, mediante a implementação de inovação de produtos e processos por parte das empresas locais, são Minas Gerais; São Paulo e Mato Grosso do Sul.

Para teste da hipótese H2, que afirma que as indústrias dos estados que implementaram leis complementares de inovação desenvolveram mais inovações que aquelas dos estados que não possuem leis complementares, realizou-se a ANOVA para medidas repetidas para os estados do Norte, visando comparar o desempenho do Estado do Pará que não possui lei complementar de inovação com o Estado do Amazonas que possui lei específica e, em seguida, a mesma análise foi realizada para comparar os estados do Sul do País a fim de comparar o desempenho do Paraná que não possui lei complementar à lei de Inovação com os demais estados que possuem a referida Lei.

O teste de Mauchly, para confirmar a hipótese de esfericidade, não apresentou resultados para a região Norte. Assim, conforme Field (2009), podem-se aplicar três correções para produzir uma razão de $\mathrm{F}$ válida, baseadas nas estimativas de esfericidade defendidas por Greenhouse e Gresser (1959) e Huynh e Feldt (1976), sendo essa primeira considerada pelo autor como uma medida de correção muito conservadora.

Os testes de Greenhouse-Geisser e Huynh-Feldt apresentaram razões de $\mathrm{F}$ iguais e em ambos os testes, o valor de $\mathrm{F}$ não é significativo, sig igual a 0,739. Assim, com base em ambas as correções, podemos aceitar que o desempenho inovativo entre os estados não é significativamente distinto.

A tabela 4 apresenta estatística descritiva da região Norte.

Tabela 4 - Estatística Descritiva Região Norte

\begin{tabular}{l|c|c|c}
\hline & Média & Desvio-padrão & N \\
\hline Amazonas (1) & 326,00 & 120,955 & 5 \\
\hline Pará (2) & 347,00 & 129,329 & 5 \\
\hline
\end{tabular}

Fonte: output do SPSS com base nos dados do PINTEC
Considerando os dados da tabela4, o estado do Pará, que não possui lei complementar de inovação, tem, em média, um maior número de empresas que inovam, considerando o período da pesquisa de 2000 a 2011.

Contudo, para comprovar se as diferenças são significativas entre os estados, aplicou-se o método estatístico de ANOVA para medidas repetidas. Tendo em vista a rejeição da hipótese de esfericidade, foram realizados testes multivariados de Pillai's trace Wilks' lambda Hotelling's trace Roy'slargest root, com significância de 0,739. Este resultado não nos permite fazer análise sobre as hipóteses.

O teste de Mauchly, para testar a hipótese de esfericidade na Região Sul, apresentou significância de 0,592; desta forma, a condição de esfericidade foi aceita apenas do teste para a Região Sul.

A tabela5 apresenta a estatística descritiva da implementação de inovação na indústriadas empresas da Região Sul.

Tabela 5 - Estatística Descritiva Região Sul

\begin{tabular}{l|c|c|c}
\hline \multicolumn{1}{c|}{ Estado } & Média & $\begin{array}{c}\text { Desvio } \\
\text { Padrão }\end{array}$ & N \\
\hline Paraná (1) & 2944,80 & 705,786 & 5 \\
\hline Santa Catarina (2) & 2789,60 & 596,759 & 5 \\
\hline Rio Grande do Sul (3) & 3519,60 & 843,158 & 5 \\
\hline
\end{tabular}

Fonte: output do SPSS com base nos dados do PINTEC.

Considerando a tabela5, percebe-se que, em média, o estado do Paraná tem maior número de empresas inovando que o estado de Santa Catarina, que possui lei complementar de inovação.

Com o objetivo de avaliar se as diferenças são significativas, foi realizado teste de efeito dentre participantes - SphericityAssumed e Huynh-Feldt com significância de 0,003,portanto podemos aceitar que os estados analisados diferem em seu desempenho inovativo.

A tabela 6 apresenta a comparação aos pares, utilizando o ajuste para comparações múltiplas de Bonferroni. 


\begin{tabular}{|c|c|c|c|c|c|c|}
\hline \multicolumn{7}{|c|}{ Tabela 6 - Comparação aos Pares Região Sul } \\
\hline \multirow{2}{*}{ (I) Sul } & \multirow{2}{*}{ (J) Sul } & \multirow{2}{*}{$\begin{array}{c}\text { Diferença } \\
\text { Média } \\
\text { (I-J) }\end{array}$} & \multirow{2}{*}{$\begin{array}{l}\text { Erro } \\
\text { Padrão }\end{array}$} & \multirow{2}{*}{ Sig. ${ }^{b}$} & \multicolumn{2}{|c|}{$\begin{array}{c}\text { Intervalo de } 95 \% \text { de confiança } \\
\text { para a diferença }{ }^{b}\end{array}$} \\
\hline & & & & & Limite Inferior & $\begin{array}{l}\text { Limite Supe- } \\
\text { rior }\end{array}$ \\
\hline \multirow[t]{2}{*}{ Paraná } & Santa Catarina & 155,200 & 137,000 & ,962 & $-387,427$ & 697,827 \\
\hline & Rio G. do Sul & $-574,800$ & 185,873 & , 109 & $-1311,002$ & 161,402 \\
\hline \multirow[t]{2}{*}{ Santa Catarina } & Paraná & $-155,200$ & 137,000 & ,962 & $-697,827$ & 387,427 \\
\hline & Rio G. do Sul & $-730,000^{*}$ & 119,854 &, 011 & $-1204,715$ & $-255,285$ \\
\hline \multirow[t]{2}{*}{ Rio G. do Sul } & Paraná & 574,800 & 185,873 &, 109 & $-161,402$ & 1311,002 \\
\hline & Santa Catarina & $730,000^{*}$ & 119,854 & 011 & 255,285 & 1204,715 \\
\hline
\end{tabular}

Fonte: output do SPSS combase nos dados do PINTEC.

Ao analisar os dados dos estados da Região Sul, observa-se, no resultado apresentado na tabela 6 , que há diferença significativa $(0,011)$ entre o desempenho inovativo das empresas dos estados do Rio Grande do Sul e Santa Catarina, contudo ambos os estados possuem leis complementares de inovação. Não se identificou, contudo, diferença significativa no desempenho do estado do Paraná em relação aos demais estados da Região Sul, o que corrobora os resultados da ANOVA de médias repetidas aplicadas aos estados da Região Norte, evidenciando, mais uma vez, que não há diferença significativa no desempenho dos estados que possuem Leis complementares de Inovação, em relação aos Estados que não possuem.

\section{CONSIDERAÇÕES FINAIS}

Este estudo investigou-se a adoção de leis de inovação complementares pelos Estados da Federação impactoua implementação de inovação nas indústrias locais, utilizando como base os dados da PINTEC apresentados nos anos de 2000, 2003, 2005, 2008 e 2011.

Por meio das evidências empíricas, foi possível constatar que 12 dos 14 Estados da Federação selecionados pela pesquisa demonstraram queda no número de empresas que implementaram inovação de produto e/ ou processo, independentemente de possuírem ou não leis complementares de inovação, no que concerne aos resultados do PINTEC 2008 e PINTEC
2011. Este resultado leva ao entendimento deque aspectos econômicos estruturais impactaram as empresas no referido período.

Destaca-se que a ausência de crescimento de inovação por parte das empresas brasileiras, no período de 2008 a 2011, pode estar relacionada à crise econômica mundial, que eclodiu a partir da crise do subprime americano, em 2008,o que pode ter minimizado o apetite do setor privado em relação ao risco relacionado aos processos de inovação, consequentemente diminuindo investimento em inovação.

Percebe-se, ainda, que oito estados da Federação, selecionados pela pesquisa, apresentaram diminuição da representatividade de empresas que implementaram inovação de produtos e processos em relação ao desempenho geral do País. Listam-se os estados: Amazonas, Pará, Bahia, Espírito Santo, Rio de Janeiro, São Paulo, Paraná e Santa Catarina.

Percebe-se, contudo, que os estados do Ceará, Pernambuco, Minas Gerais Rio Grande do Sul e Goiás passaram a ter maior representatividade de inovação no período de 2008 a 2011, tendo eles implementado leis complementares de inovação entre 2008 e 2009 . Seriam suas leis complementares mais robustas e realmente efetivas para incentivar a inovação?

$\mathrm{O}$ estudo possibilitou identificar ainda que os estados com maior participação, por meio do número de empresa locais que implementam de inovação de produtos e processos são São Paulo, Minas Gerais e Mato Grosso do 
Sul. Os três estados, soma dos têm uma representatividade de $57 \%$ de empresas que inovam no país, considerando a amostra selecionada para a pesquisa.

A análise longitudinal do desempenho inovativo dos estados não foi conclusiva significativamente para dar suporte à hipótese $\mathrm{H} 1$, logo não temos dados para quaisquer afirmações. É válido, contudo, analisar quais os aspectos que levaram os estados do Ceará, Pernambuco, Minas Gerais, Rio Grande do Sul e Goiás a aumentarem sua representatividade nas ações de inovação em relação ao desempenho dos demais estados, em um período em que a maioria dos estados apresentou decrescimento de participação.

Sugere-se especial atenção aos estados de Goiás e Pernambuco, que também apresentaram um maior número de empresas inovando, considerando o número de empresas locais pesquisadas, com crescimentos consecutivos de 2005 a 2011.

Os resultados da pesquisa, por meio da aplicação da ANOVA de medidas repetidas, para avaliar o desempenho dos estados que possuem leis complementares de inovação em relação àqueles que não possuem, identificaram que não há diferenças significativas entre o desempenho dos Estados; dessa forma, não é possível confirmar a hipótese $\mathrm{H} 2$ de que estados que possuem leis complementares de Inovação possuem desempenho inovativo significativamente superior aos estados que não implementaram lei específica.

É, contudo, uma limitação desta pesquisa, a pequena base de dados disponibilizada pelo Ministério da Ciência, Tecnologia e Inovação, mediante a pesquisa PINTEC, cujos resultados são trienais. Também se destaca como limitador o fato de a pesquisa (PINTEC) não disponibilizar os dados de todas as unidades da Federação, o que impossibilitou a ampliação da análise do desempenho.

Sugere-se, para futuras pesquisas, uma análise aprofundada das leis complementares de inovação dos estados, identificando seus aspectos positivos e falhos no incentivo à inovação local.
Propõem-se, ainda, pesquisas para identificar as razões que levaram à diminuição de ações de inovação por parte das empresas brasileiras no triênio de 2009 a 2011 e a identificação das razões que levam os estados de São Paulo, Minas Gerais e Mato Grosso do Sul a possuírem desempenho inovativo diferenciado dos demais estados, representando, os três juntos, mais de $50 \%$ das empresas que inovaram no País.

\section{REFERÊNCIAS}

ARAÚJO, N. L. Focalizando a lei de inovação. Revista Jurídica Consulex/Dialex, Brasília, v. 25, n. 73, 2007.

BARBOSA, M. A. C. "Positivismos" versus "interpretativismos": o que a Administração tem a ganhar com esta disputa? In: ANAIS DO ENCONTRO DA ANPAD, 36., 2012, Rio de Janeiro. Anais [...]. Rio de Janeiro: Anpad, 2012.

BRASIL. Portal da legislação. Governo Federal. Lei complementar. Disponível em: http:// www4.planalto.gov.br/legislacao/legislacao-1/ leis-complementares-1. Acesso em: $5 \mathrm{dez}$. 2013.

BRASIL. Constituição (1988). Constituição da República Federativa do Brasil. Brasília, DF: Senado Federal: Centro Gráfico, 1988.

BRASIL. Ministério da Ciencia, Tecnologia e Inovação. Marco Legal da Inovação. 2013. Disponível em: http://www.mct.gov.br/index. php/content/view/8477.html. Acesso em: 5 dez. 2019.

CARVALHO, M.M. Inovação: estratégias e comunidades de conhecimento. São Paulo: Editora Atlas S/A, 2009.

CAMARA, Samuel F.; BRASIL, Alexander . A coevolução entre políticas públicas/instituições e o desenvolvimento tecnológico: o caso da 
Petrobras Biocombustível. Revista de Administração Pública (Impresso), v. 49, p. 14531478, 2015.

COLLIS, J.; HUSSEY, R. Pesquisa em Administração: um guia prático para alunos de graduação e pós graduação. 2. ed. Porto Alegre: Bookman, 2005.

COOTER, R. et al. The importanceof Law in promotioninnovationandgrouwth. In: RULES For Growth: promotion, innovationandgrowththrough legal reform. Kansas City, Missouri: Erwin Marion KauffmanFudation, 2011.

DINIZ, C. C. Globalização, escalas territoriais e política tecnológica regionalizada no Brasil. Belo Horizonte: UFMG/Cedeplar, 2001.

FARIA, J. H. Dimensões da Matriz Epistemológica em estudos em administração: uma proposição. In: ANAIS DO ENCONTRO DA ANPAD, 36., 2012, Rio de Janeiro. Anais [...]. Rio de Janeiro: Anpad, 2012.

FREEMAN, C.; SOETE, L. A economia da inovação industrial. Tradução André Luiz Sica de Campos e Janaina Oliveira Pamplona da Costa. Campinas, SP: Editora da Unicamp, 2008 .

FONSECA, R. Inovação tecnológica e o papel do governo. Parcerias Estratégicas, v. 6, n. 13, p. 64-79, 2010.

GARNICA, L.A.; JUGEND, D. Estímulo à inovação em empresas de base tecnológica de pequeno porte: uma análise da lei federal brasileira de inovação. Revista da Micro e Pequena Empresa, Campo Limpo Paulista, v. 3, n. 1, p. 82-98, 2009.

GUELLEC, D. Économie de l'innovation.coll. Repères, éd. LaDécouverte, 1999.

GUELLEC, D. Les politiques de soutien à l'innovationtechnologique à l'aune de lathéorieéconomique, Economie\&prévision, n. 150, p. $95-105,2001$.

GIL, A. C. Como elaborar projetos de pesquisa. 4. ed. São Paulo: Atlas. 2007.

HADFIELD, G. Producinlaw for innovation. In: RULES For Growth: promotionginnovationandgrowththrough legal reform. Kansas City, Missouri: Erwin Marion KauffmanFudation, 2011.

HÖLZL, W.; JANGER, J. Distance to the Frontier and the Perception of Innovation Barriers across European Countries. Research Policy, v. 43, n. 4, p. 707-725, May 2014.

IBGE. Pesquisa de Inovação - PINTEC . 2019. Disponivel em: https://www.ibge.gov. br/estatisticas/multidominio/ciencia-tecnologia-e-inovacao/9141-pesquisa-de-inovacao.ht$\mathrm{ml} ?=\& \mathrm{t}=$ downloads. Acesso em: 15 dez. 2019.

ISIDRO FILHO, A.; GUIMARÃES, T. A. Conhecimento, aprendizagem e inovação em organizações: uma proposta de articulação conceitual. RAI - Revista de Administração de Inovação, São Paulo, v. 7, n. 2, p. 127-149, abr./jun. 2010.

KRETZER, J. Sistemas de Inovação: as contribuições das abordagens nacionais e regionais ou locais. Ensaios FEE, Porto Alegre, v. 30, n. 2, p. 863-892, 2009.

MATIAS -PEREIRA, J. A lei de inovação como instrumento de suporte à política industrial e tecnológica do Brasil. Rev. Cent. Ciênc. Admin., Fortaleza, v. 9, n. 1, p. 8-21, ago. 2003.

MATIAS-PEREIRA, J. Instrumentos de Desenvolvimento: um enfoque sobre a lei de inovação tecnológica do Brasil. Revista IMES Administração, v. 21, n. 60, jul./dez. 2004.

MATIAS-PEREIRA, J.; KRUGLIANSKAS, I. 
Lei de inovação tecnológica: instrumento efetivo de incentivo a inovação e a pesquisa no Brasil? Revista Gestão Industrial, Campus Ponta Grossa, Paraná, v. 2, n. 2, p. 76-88, 2006.

MINISTÉRIO DO PLANEJAMENTO, ORÇAMENTO E GESTÃO. PINTEC Pesquisa de Inovação .2013. Disponível em: http:// www.pintec.ibge.gov.br/. Acesso em: 5 dez. 2013.

MOREIRA, N. V. A. et al. A inovação tecnológica no brasil: os avanços no marco regulatório e a gestão dos fundos setoriais. Revista de Gestão USP, São Paulo, v. 14, n. especial, p. 31-44, 2007.

OECD. Eurostat. Oslo Manual 2018: Guidelines for Collecting, Reporting and Using Data on Innovation,4th Edition, The Measurement of Scientific, Technological and Innovation Activities, OECDPublishing, Paris/Eurostat, Luxembourg. 2018. https://doi.org/10.1787/ 9789264304604-en

SICSÚ, A. B.; LIMA, J. P. R. Regionalização das políticas de C\&T: concepção, ações e propostas tendo em conta o caso do Nordeste. Parcerias Estratégicas, Brasília, n. 13, p. 23-41, dez. 2001.

SCHUMPETER, J.A. Capitalismo, Socialismo e Democracia. Rio de Janeiro: Editora fundo de cultura, 1961.

SILVA FILHO, J. C. L.; BRAGA, C. S. C.; REBOUCAS, S. M. D. P. Percepção de Obstáculos à Inovação na Indústria Brasileira de Transformação. International Journal Of Innovation, v. 5, p. 114, 2017.

STAL, E; FUJINO, A. A lei de inovação como instrumento de suporte à política industrial e tecnológica do Brasil. Caderno de Pós-Graduação - administração, São Paulo, v. 4, n. 1, Especial RAI, p. 269-283, 2005.

STAUB, E. Desafios estratégicos em ciência, tecnologia e inovação. Brasília: IEDI, 2001. Disponível em: http://www.iedi.org.br. Acesso em: 27 dez. 2013. 